\title{
Determination of the wind speed and direction by means of fluidic-domain signal processing
}

\author{
P. Bruschi, and M. Piotto
}

\begin{abstract}
This paper presents an analytical model for a recently introduced class of 2-D directional anemometers based on fluidic structures capable of averaging the differential pressure developed by the wind across distinct diameters of the transverse cross-section of a single cylinder. In previous works, it was found that performing the average over a proper set of diameters produces a differential pressure that depends on the wind direction according to a cosine law, allowing simple direction estimation. This fact, which was not investigated in previous articles, is explained in this paper taking into account symmetry and angular spectral properties of the pressure distribution. Besides analyzing previously proposed devices, this paper introduces several new configurations, which are classified according to the type of average and number of diameters involved. Comparison of the estimated performances with the experimental results obtained in earlier works clearly shows that prototypes proposed so far were far from achieving the best theoretical accuracy, suggesting that significant improvements can be obtained by re-design of the fluidic structures.
\end{abstract}

Index Terms-2-D anemometer, fluidic-domain signal processing, cylindrical pressure probe, pressure probe theory.

\section{INTRODUCTION}

$\mathrm{M}$ EASUREMENT of the wind velocity and direction has played a key role for a long time in many different scenarios, such as meteorological monitoring and forecast, navigation, sport disciplines (sailing, track and field, golf etc.), and landing/takeoff activities in airfields.

Recently, innovative applications of anemometers are being suggested by the introduction of autonomous vehicles. Detection of the wind velocity and direction is essential for maintaining the correct pitch angle and relative airspeed of fixed wing drones [1] and for sail adjusting in autonomous sailboats [2]. Algorithms that make use of directional anemometers have been proposed to allow terrestrial or aerial unmanned vehicles to find and track pollutant sources [3]. Anemometers designed for these applications have to be small and inexpensive, use low power and be free from moving parts, to avoid sensitivity to inertial forces. Ultrasonic anemometers [4] currently represent the option that best meets

Submitted on October 21, 2017.

M. Piotto is with the CNR-IEIIT institute, 56122 Pisa, Italy. (e-mail: massimo.piotto@ieiit.cnr.it).

P. Bruschi is with the Dipartimento di Ingegneria dell'Informazione, of the University of Pisa, 56122 Pisa, Italy (e-mail:p.bruschi@iet.unipi.it). He is also an associate researcher of the CNR-IEIIT institute these requirements. However, their cost is comparable to that of a complete small autonomous vehicle, making their adoption not convenient. Furthermore, the power consumption of currently available ultrasonic anemometers is at least several hundred $\mathrm{mW}$, which is a significant fraction of the power budget of a small robot. Development of low-cost ultrasonic anemometers [5] was proposed, but their accuracy is not adequate for critical operations, such as sail settings in autonomous sailboats.

Examples of small robots employing thermal anemometers have been proposed [6]. Thermal sensors are relatively compact and maintain an excellent sensitivity even at reduced pressures [7]. The main drawbacks are high power consumption and small bandwidth [8]. A significant improvement can be reached by using MEMS (Micro ElectroMechanical System) technologies to reduce thermal masses and increase thermal insulation $[9,10]$. However, typical MEMS microstructures are fragile and their response is prone to be altered by even single dust particles or water droplets.

Another popular solution is represented by pressure probes. The simplest pressure probe is the well-known Pitot tube, which measures only the absolute value of the wind velocity. Multi-hole pressure probes [11] provide also information on the wind direction by comparing several pressure values taken at different points on the outer surface of a bluff or streamlined body exposed to the wind. The so-called "Cobra" probes [12-15], based on a faceted head, are characterized by reduced direction ranges. Extension to a full $360^{\circ} 2-\mathrm{D}$ range or to full 3-D detection capability has been proposed by multi-point measurement of the pressure distribution around cylinders $[16,17]$ and spheres $[18-20]$. In these devices, the pressure is sampled by several holes drilled into the body surface, connected with pipes to independent pressure transducers. Therefore, the complete wind sensor turns out to be relatively large and power demanding. In addition, complex procedures are required to calibrate the semi-empirical algorithms used to estimate the quantities of interest [21]. These aspects conflict with the typical requirements for sensors to be placed on board small autonomous vehicles.

An ideal solution would be designing a probe with a shape and configuration of holes capable of producing a differential pressure that exhibits a cosine dependence on the angle formed by the wind with respect to a reference axis. Two orthogonal probes with such a response would produce two pressure signals proportional to the cosine and sine of the wind direction (angle). Then, the angle could be calculated by 
means of a simple arctangent operation. In addition, the sum of the squares of the two pressures would depend only on the wind velocity, which could be simply estimated from a 1dimensional calibration curve.

In 2009 [22], we showed that a differential pressure that exhibits a cosine dependence on the wind direction could be obtained by averaging several pressure differences taken across distinct diameters of the transverse cross section of a single cylinder (diametric pressures). This is not trivial, since the original dependence of diametric pressures is far from being sinusoidal. Using this principle, indicated here with DPA (Diametric Pressure Averaging), we built an innovative 2-D pressure probe that used a proper pipe structure to calculate the required average in the fluidic domain. The two orthogonal section required to calculate the wind direction and velocity were carved inside the same cylinder body. Overall, only two differential pressure sensors are required, while calculations are simple and straightforward. Operation at wind velocities as low as $1 \mathrm{~m} / \mathrm{s}$ and below was obtained by measuring the small pressure differences (order of $1 \mathrm{~Pa}$ ) by means of high-resolution MEMS sensors exploiting detection of the flow rates induced by the pressure to be measured through capillary pipes. Prototypes proposed so far use custom-made MEMS sensors [22-24], which can be replaced by inexpensive commercial devices (e.g. Sensirion SDP800 differential pressure sensors [25]) at only the price of moderate size increase. In this way, anemometers that combine overall dimensions of a few centimeters with a much lower cost and power-consumption than ultrasonic anemometers can be obtained.

We have demonstrated the effectiveness of different fluidic structures, performing three-diameter [22] and five-diameter [23] arithmetic DPA, and seven-diameter weighted DPA [24]. In all cases, the main parameters (e.g. the angles between the diameters) were found by means of an exhaustive numerical algorithm that minimizes the deviation from the target cosine law. Such optimization, performed at a single wind velocity, was maintained across a large velocity range (more than a decade) in spite of apparent changes in the shape of the pressure distribution. This was regarded as an unexpected result, and a theoretical explanation was missing.

The aim of this work is to derive an analytical justification of the DPA method from the characteristics of the diametric pressures. The paper is organized as follows: Sect. II introduces the main quantities and conventions, showing also the symmetries and the spectral properties of the function that relates the diametric pressure to the angle formed by the diameter with the wind direction. The role of higher order harmonics in producing the observed deviation from the cosine law is highlighted. In Sect. III, it will be shown that the contribution of higher order harmonics can be reduced by a proper average of distinct diametric pressures (DPA), leading to excellent approximations of the cosine dependence. Two possible DPA approaches to the approximation of the cosine dependence are examined. Implementation of the two approaches with fluidic structures is analyzed in Sect. IV while comparison of the proposed configurations in terms of theoretical accuracy is presented in Sect. V. Finally, in Sect. VI the analytical predictions of the proposed model are compared with experimental results described in our previous works [22-24] concerning prototypes implementing three different DPA configurations.

\section{ANALYSIS OF THE PRESSURE DISTRIBUTION AROUND A CYLINDER}

The main conventions used in this work are illustrated in Fig.1, representing a cross-section of the cylinder with a fixed reference axis $x$. The wind vector $v$, lying in the same plane as the cylinder cross-section, forms an angle $\theta$ with axis $x$.

A point on the cylinder surface is determined by the angle $\phi$ formed by the radius passing from the point and the reference axis. For a non-zero wind velocity, pressure differences with respect to the undisturbed flow develop in the air around the cylinder. The value assumed by the pressure difference at a point on the cylinder surface is indicated with $p_{S}(\phi, \theta, u)$, to emphasize dependence upon position $(\phi)$, wind direction $(\theta)$ and wind magnitude $(u)$.

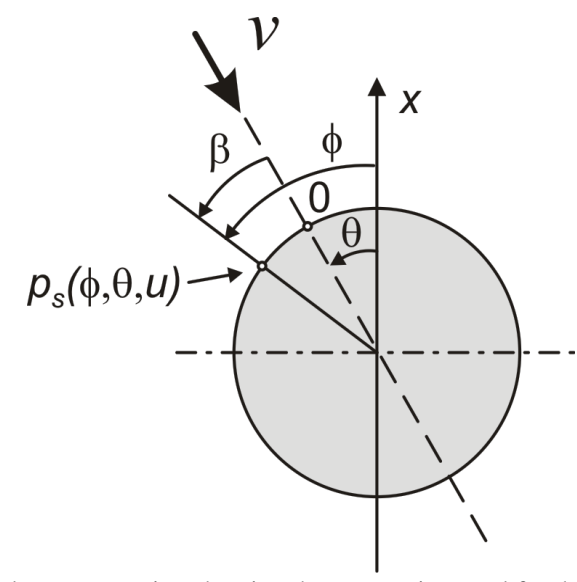

Fig.1. Cylinder cross-section showing the convention used for the quantities of interest. The wind velocity vector and the stagnation point are indicated with "v" and "0", respectively.

In order to reduce the number of variables, it is convenient to consider that, for a given wind velocity, the surface pressure depends only on the angular distance $(\beta=\phi-\theta)$ from the stagnation point, indicated with " 0 " in Fig.1. We will use the notation $p_{0}(\beta, u)$ to indicate the function that relates $\beta$ and $u$ to the surface pressure. It can be easily shown that the following transformation holds:

$$
p_{S}(\phi, \theta, u)=p_{0}(\phi-\theta, u)=p_{0}(\beta, u)
$$

For the analysis that follows, the most relevant quantity is the "diametric pressure" defined as the pressure difference between two diametrically opposite points. Again, we can identify points on the surface with the angular distance either from the fixed reference axis $(x)$ or from the stagnation point. Accordingly, the respective expressions for the diametric pressure are: 


$$
\begin{aligned}
& p_{D S}(\phi, \theta, u) \equiv p_{S}(\phi, \theta, u)-p_{S}(\phi+\pi, \theta, u) \\
& p_{D 0}(\beta, u) \equiv p_{0}(\beta, u)-p_{0}(\beta+\pi, u)
\end{aligned}
$$

Obviously, the transformation law given by (1) is valid also for the diametric pressures, i.e.:

$$
p_{D S}(\phi, \theta, u)=p_{D 0}(\phi-\theta, u)
$$

Fig. 2 (a) shows the pressure distribution on a cylinder surface as a function of angle $\beta$ for different Reynolds numbers defined by:

$$
\operatorname{Re}=\frac{\rho u D}{\mu},
$$

where $u$ is the undisturbed wind velocity (magnitude of vector $v), \rho$ and $\mu$ are the air density and viscosity, respectively, whereas $D$ is the cylinder diameter. These data have been obtained by precisely digitizing a set of experimental curves reported in [26]. Interpolation has been performed using cubic splines. As customary, pressure data are normalized to the dynamic pressure $\left(\rho u^{2} / 2\right)$. From the data of Fig.2 (a) we have calculated the corresponding diametric pressures, shown in Fig. 2 (b).
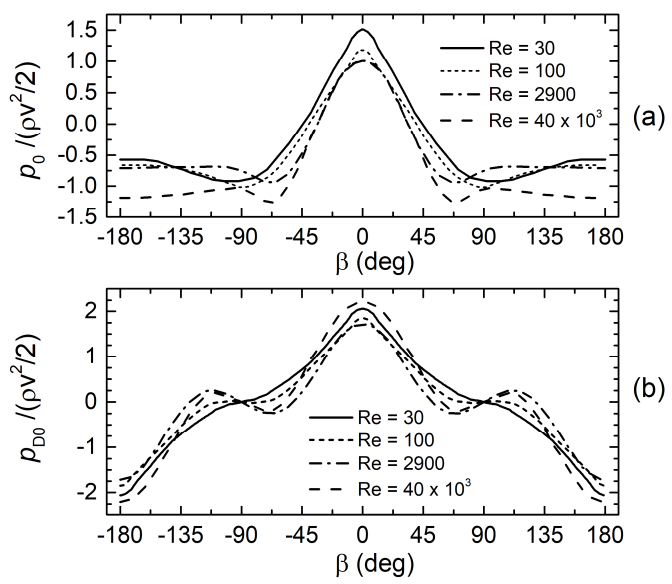

Fig.2. Normalized pressure (a) and diametric pressure (b) around the cylinder lateral surface as a function of $\beta$ for different Reynolds numbers $(\mathrm{Re})$.

The first question is whether it is possible to calculate the wind direction $(\theta)$ and velocity $(\mathrm{u})$ from the knowledge of only two diametric pressures, picked up along two orthogonal axis, namely $x$ and $y$.

Considering that the $x$ axis is placed at a distance $\beta=-\theta$ from the stagnation point, then the diametric pressure along $x$, $p_{D X}(\theta, u)$, is equal to $p_{D 0}(-\theta, u)=p_{D o}(\theta, u)$. Similarly, the pressure along $\mathrm{y}$ is given by $p_{D Y}(\theta, u)=p_{D 0}(\pi / 2-\theta, u)$ $=p_{D 0}(\theta-\pi / 2, u)$. Due to the non-monotonic behavior of the $\mathrm{p}_{D 0}(\theta, u)$ function in the interval $0-\pi$ for Re $>100$, determination of $\theta$ and $u$ from measurement of only two orthogonal diametric pressures is not possible [22]. Solutions that rely on monitoring three diametric pressures have been envisioned [27], although complex multi-dimensional look-up tables are needed. Estimation of wind speed and direction from four diametric pressures was demonstrated in [28] by means of a prototype that uses four differential pressure sensors.

The task would be strongly facilitated if $p_{D o}(\beta, u)$ were proportional to $\cos (\beta)$. The fact that this does not happen means that harmonics of higher order than the fundamental are present in the spectrum of $p_{D 0}(\beta, u)$ calculated over the interval $[-\pi, \pi]$. The result of angular spectral analysis of the $p_{D o}(\beta, u)$ with respect to variable $\beta$ is shown in Fig.3. The figure displays the minimum and maximum normalized magnitude of each harmonic component across the following set of Re numbers: $30,100,250,1240,2900,8500$ and $40 \times 10^{3}$. Due to the half-wave symmetry of $p_{D o}(\beta, u)$, only odd harmonics are present. It is apparent that the difference from a pure sinusoidal function mainly derives from the relatively large third harmonic component.

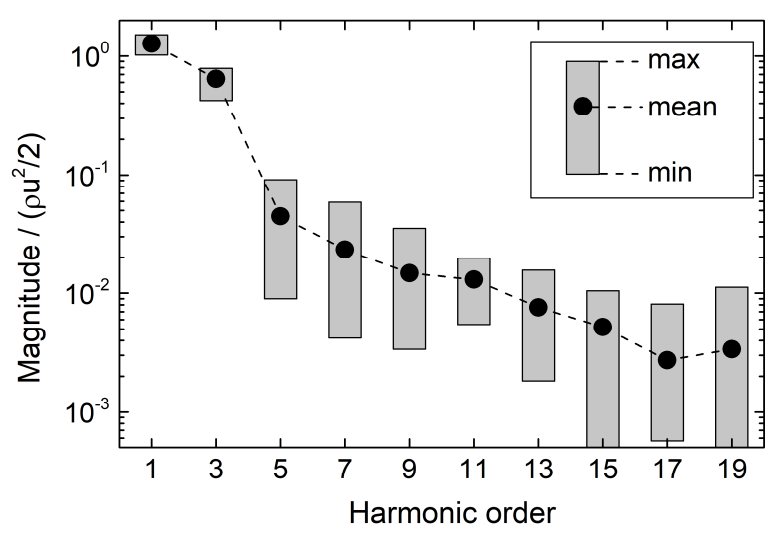

Fig.3. Angular spectral analysis of the diametric pressure $p_{D o}(\beta, u)$ with respect to variable $\beta$ over the interval $[-\pi, \pi]$. The minimum and maximum values of each harmonic magnitude refer to a Re range from 30 to $40 \times 10^{3}$. The magnitude of the harmonic components is normalized to $\rho u^{2} / 2$.

\section{COSINE APPROXIMATION BY MEANS OF LINEAR COMBINATION OF DIAMETRIC PRESSURES}

\section{A. General case}

A way to obtain a differential pressure that approximate the desired $\cos (\theta)$ dependence is combining several diametric pressures in order to reduce the content of higher order harmonics. The diameters taken into consideration in this linear combination form a configuration that is symmetrical with respect to axis $x$, from which they are separated by the following $2 N+1$ angles:

$$
\begin{aligned}
& \left\{\phi_{0}, \pm \phi_{1}, \pm \phi_{2}, \ldots \ldots \pm \phi_{N}\right\} \\
& \text { with } \phi_{0}=0 \text { and } \phi_{i} \leq \pi / 2 \text { for } i \in[1, N]
\end{aligned}
$$

Summing up the diametric pressures taken across the diameters identified by (5), the following differential pressure can be obtained:

$$
p_{X}(\theta, u)=\sum_{i=-N}^{N} w_{i} p_{D S}\left(\phi_{i}, \theta, u\right)
$$

where $w_{\mathrm{i}}$ are arbitrary positive weights such that $w_{-\mathrm{i}}=w_{\mathrm{i}}$ and $\phi_{-\mathrm{i}}=-\phi_{\mathrm{i}}$. Using (3), we obtain the equivalent expression: 


$$
p_{X}(\theta, u)=\sum_{i=-N}^{N} w_{i} p_{D 0}\left(\phi_{i}-\theta, u\right)
$$

It is convenient to represent the dependence of $p_{D O}(\theta, u)$ and $p_{X}(\theta, u)$ on $\theta$ with a Fourier series:

$$
\begin{aligned}
& p_{D 0}(\theta, u)=\sum_{k=1,3,5 \ldots}^{\infty} A_{k}(u) \cos (k \theta) \\
& p_{X}(\theta, u)=\sum_{k=1,3,5 \ldots}^{\infty} B_{k}(u) \cos (k \theta)
\end{aligned}
$$

where $A_{k}(u)$ and $B_{k}(u)$ indicates coefficients that depend on the wind velocity $u$. Note that only odd cosine components are present due to the even0 half-wave symmetry of $p_{D o}(\theta, u)$, which can be easily extended to $p_{X}(\theta, u)$, from its definition. By means of simple passages, it is possible to relate coefficients $B_{k}$ to coefficients $A_{k}$ :

$$
B_{k}(u)=A_{k}(u) \sum_{i=-N}^{N} w_{i} \cos \left(k \phi_{i}\right)
$$

Notice that the fundamental component, given by:

$$
B_{1}=A_{1}(u) \sum_{i=-N}^{N} w_{i} \cos \left(\phi_{i}\right)
$$

is always positive due to (5) and the condition $w_{\mathrm{i}}>0$.

With a proper combination of weights $w_{\mathrm{i}}$ and angles $\phi_{\mathrm{i}}$ it is possible, at least in principle, to make $p_{X}(\theta, u)$ approximate a cosine law. We will consider two particular cases: (i) arbitrary angles $\phi_{i}$ with uniform weights (UW) and (ii) uniform diameter spacing (UDS) with arbitrary weights.

\section{B. Case 1: Uniform weights (UW)}

In the case of uniform weights, it is possible to cancel all nonfundamental harmonics up to the $(2 N+1)$-th one by imposing $B_{\mathrm{k}}=0$ for $k=3,5, \ldots, 2 N-1$ in (10). The $N$ unknowns to be found are angles $\phi_{\mathrm{i}}$ for $1 \leq i \leq \mathrm{N}$. It is worth recalling that all even harmonics are already zero for the mentioned symmetry properties. The higher $N$, the higher the number of harmonics that can be cancelled, and then, the better is the cosine approximation. However, large $N$ values result in increased system complexity, since the number of diametric pressures involved is equal to $2 N+1$. Recalling that $\phi_{\mathrm{i}}=-\phi_{-\mathrm{i}}$, and $\phi_{0}=0$, and imposing $B_{k}=0$ for $k>1$, equations (10) can be re-arranged to obtain the following equation set:

$$
1+2 \sum_{i=1}^{N} \cos \left(k \phi_{i}\right)=0 \quad \text { for } k=3,5, \ldots, 2 N+1
$$

Since the larger non-fundamental harmonic component is the third one, even with $N=1$ it is possible to obtain a good approximation of the cosine function. In that case, equation set (12) reduces to the following single equation, which should be satisfied to cancel the third harmonics:

$$
1+2 \cos \left(3 \phi_{1}\right)=0
$$

Equation (13) has two exact solutions in the $[0, \pi / 2]$ interval: $\phi_{1 \mathrm{a}}=40^{\circ}$ and $\phi_{1 \mathrm{~b}}=80^{\circ}$. For $N>1$, equation set (12) should be solved by means of numerical procedure. Table I shows the optimal angles for $N$ in the range 1-5, determined by numerical solution of equation set (12). For each $N$ value, two solutions, marked with "a" and "b", are reported in Table I. Solutions " $b$ " differ from "a" ones for having the largest angle of the set, $\phi_{\mathrm{N}}$, lying in the interval $80^{\circ}-90^{\circ}$.

TABLE I. OPTIMAL ANGLES FOR THE UW CONFIGURATIONS

\begin{tabular}{|l|l|l|l|l|l|l|}
\hline $\mathrm{N}$ & Solution & $\phi_{1}$ & $\phi_{2}$ & $\phi_{3}$ & $\phi_{4}$ & $\phi_{5}$ \\
\hline \multirow{3}{*}{1} & $\mathrm{a}$ & $40^{\circ}$ & - & - & - & \\
\hline & $\mathrm{b}$ & 80 & & & & \\
\hline \multirow{2}{*}{2} & $\mathrm{a}$ & $21.8^{\circ}$ & $52.0^{\circ}$ & - & - & \\
\hline & $\mathrm{b}$ & 33.9 & 84.2 & & & \\
\hline \multirow{3}{*}{3} & $\mathrm{a}$ & $17.0^{\circ}$ & $32.5^{\circ}$ & 58.3 & - & \\
\hline & $\mathrm{b}$ & 20.7 & 46.2 & 85.8 & & \\
\hline \multirow{3}{*}{4} & $\mathrm{a}$ & 11.3 & 27.7 & 39.0 & 62.4 & \\
\hline & $\mathrm{b}$ & $15.5^{\circ}$ & $31.4^{\circ}$ & 53.1 & 86.6 & \\
\hline 5 & $\mathrm{a}$ & 14.1 & 16.4 & 35.5 & 43.3 & 62.5 \\
\hline & $\mathrm{b}$ & 11.8 & 25.2 & 38.4 & 57.6 & 87.2 \\
\hline
\end{tabular}

C. Case 2: Uniform diameter spacing (UDS)

With the second approach (UDS), the weights are not uniform while the angles are uniformly distributed between 0 and $\pi / 2$. Introducing an integer $M$, angles $\phi_{\mathrm{i}}$ can be expressed as follows:

$\phi_{i}=i \frac{\pi}{2 M} \quad$ where $i=0,1, \ldots, M$

Considering that, as in the general case, $\phi_{-\mathrm{i}}=-\phi_{\mathrm{i}}$ and $w_{-\mathrm{i}}=w_{\mathrm{i}}$, Eqn. (7) becomes:

$$
p_{X}(\theta, u)=\sum_{i=-M}^{M} w_{i} p_{D 0}\left(i \frac{\pi}{2 M}-\theta, u\right)
$$

Let us focus on the following choice for the weights:

$w_{i}=w_{0} \cos \left(\phi_{i}\right)=w_{0} \cos \left(i \frac{\pi}{2 M}\right)$,

where $\mathrm{w}_{0}$ is the weight of the central diametric pressure $(i=0)$. Substituting (16) into (15) we get:

$$
p_{X}(\theta, u)=w_{0} \sum_{i=-M}^{M} p_{D 0}\left(i \frac{\pi}{2 M}-\theta, u\right) \cos \left(i \frac{\pi}{2 M}\right)
$$

It can be easily shown that the sum in (17) represents an approximation of the $\cos (\phi) p_{D 0}(\phi-\theta, \mathrm{u})$ integral over the $[-\pi / 2, \pi / 2]$ interval, calculated over a set of discrete points separated by $\pi / 2 \mathrm{M}$ increments. More precisely:

$p_{X}(\theta, u) \cong w_{0} \frac{2 M}{\pi} \int_{-\pi / 2}^{\pi / 2} p_{D 0}(\phi-\theta, u) \cos (\phi) d \phi$

Using the Fourier series expansion of $p_{D 0}$ given by (8):

$$
p_{X}(\theta, u) \cong w_{0} \frac{2 M}{\pi} \int_{-\pi / 2}^{\pi / 2}\left\{\sum_{k=1,3,5 \ldots}^{\infty} A_{k}(u) \cos [k \phi-k \theta]\right\} \cos (\phi) d \phi
$$

With tedious but elementary transformations, it is possible to demonstrate that the integral in (19) is exactly equal to 
$(\pi / 2) A_{1}(u) \cos (\theta)$, so that (19) becomes:

$$
p_{X}(\theta, u) \cong w_{0} M A_{1}(u) \cos (\theta)
$$

In this way, we have obtained the desired proportionality to $\cos (\theta)$. Clearly, the higher the number of points $(2 M+1)$, the better the approximation of the integral and then of the cosine law. The addends for $i= \pm M$, corresponding to $\phi_{\mathrm{i}}= \pm \pi / 2$ do not give contributions since their weights, given by (16), are zero. Then, (17) can be rewritten as:

$$
p_{X}(\theta, u)=w_{0} \sum_{i=-N}^{N} p_{D 0}\left(i \frac{\pi}{2(N+1)}-\theta, u\right) \cos \left(i \frac{\pi}{2(N+1)}\right)
$$

where $N=M-1$ plays the same role as in the UW case, since the actual number of diametric pressure to be combined is $2 N+1$. It is possible to derive (20) from (18) also with more intuitive arguments. Indeed, the integral in (18) coincides with the expression of the cosine component of the first harmonics of function $p_{D 0}(\phi-\theta, u)$. Differently from the general expressions of the Fourier series components, the integral can be calculated over half period $(\pi)$ instead of the whole period $(2 \pi)$, due to the half-wave symmetry of $p_{D o}(\phi-\theta, u)$. Since $p_{D o}(\phi-\theta, u)$ is obtained by shifting $p_{D O}(\phi, u)$ by $\theta$, it loses the even symmetry of $p_{D o}(\phi, u)$ and both cosine and sine components will be present in the $p_{D O}(\phi-\theta, u)$ Fourier expansion. In particular, the coefficient of the fundamental cosine component, which is extracted by (18), is just proportional to $A_{1} \cos (\theta)$, proving (20).

\section{LINEAR COMBINATION OF PRESSURES IN THE FLUIDIC DOMAIN}

\section{A. Description and advantages of the fluidic approach}

A straightforward implementation of the general approach given by (6) is using $2 N+1$ differential pressure sensors to detect the corresponding diametric pressures and then combining the output signals in the electrical domain. This solution offers the maximum flexibility, since it allows postmanufacturing tuning of the weights in order to compensate for fabrication tolerances. On the other hand, this approach requires a large number of sensors. Considering that two orthogonal sections (" $X$ " and " $Y$ ") are required for the determination of the wind vector on a plane, the total number of differential pressure sensors for a 2-D anemometer is $2(2 N+1)$. Even in the case $N=1$, six pressure sensors are required. Furthermore, sensors belonging to the same section should have closely matched sensitivities, which can be obtained only with individual sensor calibration.

An alternative solution is performing the linear combination in the fluidic domain, so that only one differential pressure sensor per section is required. The principle is illustrated by Fig. 4(a), showing a cross-section of the cylinder where two symmetrical sets of channels connect cavities $\mathrm{H}_{1}$ and $\mathrm{H}_{2}$ with the outer surface. The channels reach the cylinder surface at angular positions $\phi_{\mathrm{i}}$ (see the figure). The example in Fig. 4(a) refers to the particular case $N=2$; configurations for different $N$ values are conceptually similar.
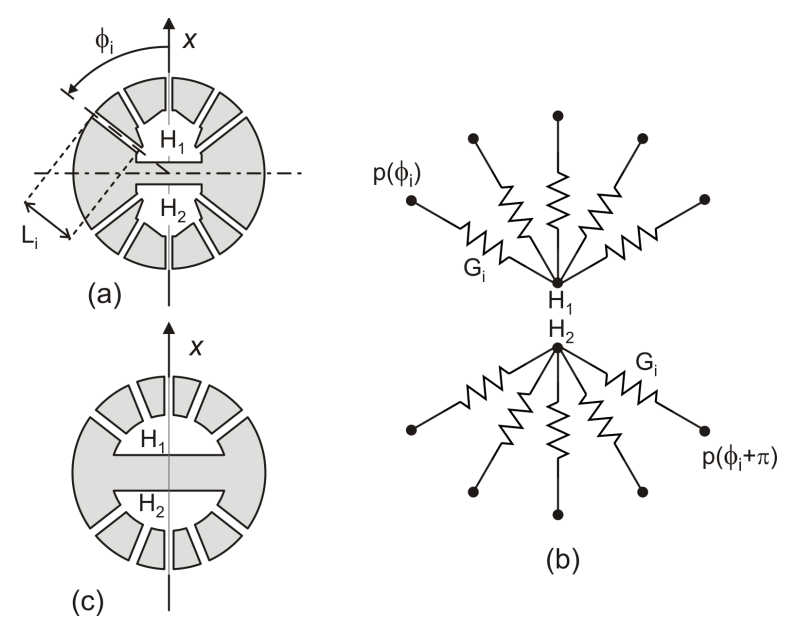

(b)

Fig.4. (a) Cylinder cross-section showing an example of fluidic structure implementing a linear combination of surface pressures with non-uniform weights. (b) Electrical equivalent network of the fluidic structure. (c) Uniformweight configuration.

Considering micro-channels with maximum diameter of a few millimeters, it is reasonable to assume that the flow through the channels is laminar. In these conditions, the relationship between pressure drop and flow rate is linear, allowing the use of the equivalent electrical circuit [29] shown in Fig. 4(b) to represent the fluidic structure. In this equivalence, pressures are voltages and flow rates are currents. The fluidic conductance of a channel $(\mathrm{G})$ is defined as the ratio of the mass flow rate $(\mathrm{Q})$ over the pressure drop across the channel $(\Delta P)$. From the well-known Hagen-Poiseuille formula for laminar flow, it is possible to derive the following expression for $\mathrm{G}[22]$ :

$$
G=\frac{Q}{\Delta P}=\rho \frac{\pi}{128} \frac{D_{e}^{4}}{\mu L},
$$

where $L$ is the channel length and $D_{e}$ is the equivalent channel diameter, defined as $D_{e}=4 A / P$, with $A$ and $P$ indicating the area and perimeter of the channel cross-section, respectively.

Solving the simple electrical network of Fig. 4 (b), the following expression can be found for the pressure difference between the two cavities:

$p_{H 1}-p_{H 2}=\sum_{i=-N}^{N} \frac{G_{i}}{G_{T}} p_{S}\left(\phi_{i}, \theta, u\right)-\sum_{i=-N}^{N} \frac{G_{i}}{G_{T}} p_{S}\left(\phi_{i}+\pi, \theta, u\right)$

where $G_{i}$ is the fluidic conductance of channel at angle $\phi_{\mathrm{i}}$. and $G_{T}$ is the sum of all $G_{i}$, that is:

$$
G_{T}=\sum_{i=-N}^{N} G_{i}
$$

Simple rearrangement of the terms in (23) leads to:

$p_{H 1}-p_{H 2}=\sum_{i=-N}^{N} \frac{G_{i}}{G_{T}} p_{D S}\left(\phi_{i}, \theta, u\right)$

which is equivalent to (6) with: 


$$
w_{i}=\frac{G_{i}}{G_{T}}
$$

In this way, we have demonstrated that the fluidic structure of Fig. 4 (a) is capable of producing a differential pressure $\left(p_{H 1}-p_{H 2}\right)$ which is equal to the required combination of diametric pressures expressed by (6).

\section{B. UW-DPA obtained with uniform channel characteristics}

If the channels are identical (same length and crosssections), as shown in Fig.4(c), all $G_{i}$ are nominally equal and the fluidic structure implements the UW case, examined in Sect. III-B. Considering (26), all weights assume the value $1 /(2 N+1)$. If angles $\phi_{i}$ are chosen according to Table I, the differential pressure $p_{H 1}-p_{H 2}$ follows a nearly cosine dependence on $\theta$. The maximum magnitude of the pressure, assumed for $\theta=0$ and $\pi$, coincides with coefficient $B_{1}$, which depends on $A_{1}$ through (11). Note that $A_{1}$ is the magnitude of the fundamental component of the Fourier series expansion of the diametric pressure $p_{D o}(\theta, u)$. Considering Fig.3, the $A_{1} / \rho u^{2} / 2$ ratio does not vary much when $\mathrm{Re}$ is swept across two orders of magnitude (from 30 to $40 \times 10^{3}$ ). Thus, $A_{1}$ can be considered nearly proportional to $\rho u^{2} / 2$. The $B_{1} / A_{1}$ ratio is given in Table II for all solutions represented in Table I. It can be observed that this ratio is higher for solutions "a" than for solution " $b$ ". This means that, for a given wind velocity, solutions "a" are capable of producing a larger differential pressure and are then advantageous for high sensitivity devices. Consequently, in the rest of this paper, only solution "a" will be analyzed.

TABLE II. $\mathrm{B}_{1} / \mathrm{A}_{1}$ RATIOS AS A FUNCTION OF N FOR THE UW SOLUTIONS

\begin{tabular}{|l|l|l|l|l|l|}
\hline$N$ & 1 & 2 & 3 & 4 & 5 \\
\hline$B_{1} / A_{1}$ (Solution a) & 0.844 & 0.817 & 0.807 & 0.801 & 0.798 \\
\hline$B_{1} / A_{1}$ (Solution b) & 0.449 & 0.572 & 0.629 & 0.661 & 0.682 \\
\hline
\end{tabular}

\section{UDS-DPA obtained with non-uniform channel lengths.}

Non-uniform channels, i.e. channels with different lengths and/or cross-sections are required to implement the UDS case examined in Sect. III-C, requiring different weights for different diameters. Tuning of $L$ and cross-sections (i.e. $D_{e}$ ) allows modulation of the conductance of the individual channels, according to (22). In order to obtain weights that satisfy (16), the channel conductance must be given by:

$$
G_{i}=G_{0} \cos \left(\phi_{i}\right),
$$

where $G_{0}$ is the conductance of the central channel (angle $\left.\phi_{\mathrm{i}}=0\right)$.

In the rest of this paper, we assume that the fluidic conductance is modulated acting on $L$. Changing channel cross-section is clearly also a viable option. Taking into account (22) and (27), the length $L_{i}$ of the channel at angle $\phi_{\mathrm{i}}$ should be given by:

$$
L_{i}=\frac{L_{0}}{\cos \left(\phi_{i}\right)}
$$

where $L_{0}$ is the length of the central channel.

As for the case of uniform channels, it is interesting to consider the ratio $B_{1} / A_{1}$. From (14), (24) and (27):

$$
G_{T}=G_{0} \sum_{i=-N}^{N} \cos \left(\frac{\pi}{2 M} i\right) \cong G_{0} \frac{2 M}{\pi} \int_{-\pi / 2}^{\pi / 2} \cos (\phi) d \phi
$$

where the approximation of the sum with the integral is made following the same considerations used for (18). Calculating the integral and using (26) with $i=0$ it is possible to find $\mathrm{w}_{0}$, which turns out to be approximated by: $w_{0}=\pi / 4 M$. Substituting this value into (20), and recalling the meaning of $B_{1}$, we find the approximation:

$$
B_{1} \cong \frac{\pi}{4} A_{1},
$$

The expression in (30) should be regarded as an asymptotic limit, which is reached for $N$ that tends to infinity and the sums in (17) and (29) converge to the corresponding integrals. The exact values of the $B_{1} / A_{1}$ ratio are reported in Table III. Consistence with the asymptotic value $\pi / 4$ is within nearly $5 \%$ even for $N=1$.

TABLE III. B $\mathrm{B}_{1} / \mathrm{A}_{1}$ RATIOS AS A FUNCTION OF N FOR THE UDS SOLUTIONS.

\begin{tabular}{|l|l|l|l|l|l|}
\hline$N$ & 1 & 2 & 3 & 4 & 5 \\
\hline$B_{1} / A_{1}$ & 0.828 & 0.804 & 0.796 & 0.792 & 0.790 \\
\hline
\end{tabular}

\section{PERFORMANCE ESTIMATION}

According to the cases studied so far, a fluidic structure can be classified as UW (Uniform Weights) or UDS (Uniform Diameter Spacing). For each type, number $N$ defines the complexity of the channel configuration, since the number of different diametric pressures involved is equal to $2 N+1$, and the total number of channels that connect cavities $\mathrm{H}_{1}$ and $\mathrm{H}_{2}$ to the outer surface are just twice this number. In order to determine the optimal structure for a given set of specifications, it is important to investigate the impact of these parameters on the device performances. Equation (6) has been implemented by means of the spline interpolation already mentioned in section II. A program using the python-scipy scientific modules [30] have been used to automate the calculations. A few significant results are shown in Figs. 5-7. Figure 5 shows the result of the cosine approximation obtained for $\mathrm{Re}=100$ with UW and UDS configuration in the simplest case of $N=1$. The ideal cosine behavior is shown to facilitate comparison.

It is apparent that both the UW and UDS configurations do not produce a good approximation of the cosine curve for $N=1$. The quality of the approximation was found to depend significantly on the Reynolds number. The case $\mathrm{Re}=100$, shown in Fig.5 is the worst that we have analyzed. A much better approximation of the cosine curve can be obtained even with $N=1$ at different Reynolds numbers, such as $\mathrm{Re}=2900$ shown in Fig. 6. 


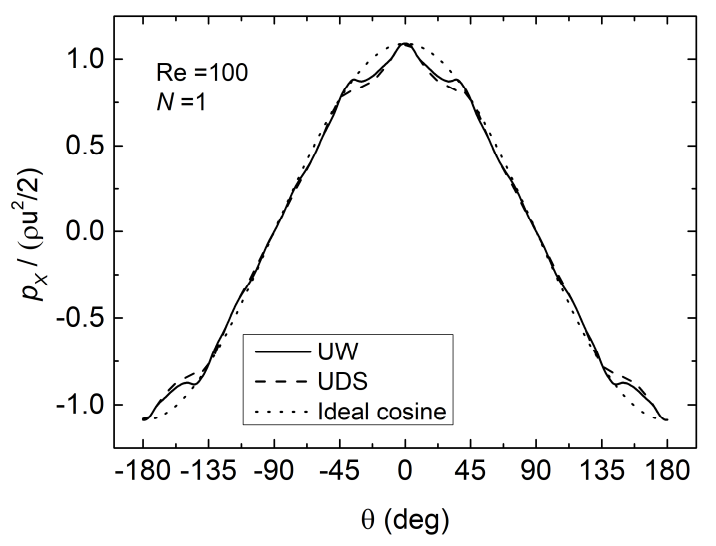

Fig.5. Output differential pressure $\left(p_{X}\right)$ at $\mathrm{Re}=100$, normalized to the dynamic pressure as a function of the wind direction for the UW and UDS solutions with $N=1$. Noticeable departure from the ideal cosine function is visible.

A good approximation throughout the whole explored Re range (from 30 to $40 \times 10^{3}$ ) can be obtained with a higher $N$ value. For example, Fig. 7 shows that an excellent approximation of the cosine dependence can be obtained at $\mathrm{Re}=100$ with $N=3$ for both UW and UCS configurations.

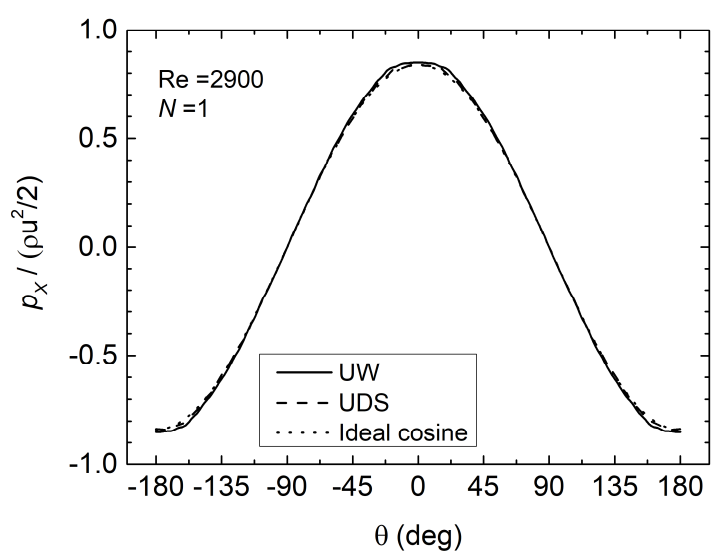

Fig.6. Output differential pressure $\left(p_{X}\right)$ at $\mathrm{Re}=2900$, normalized to the dynamic pressure as a function of the wind direction for the UW and UDS solutions with $N=1$.

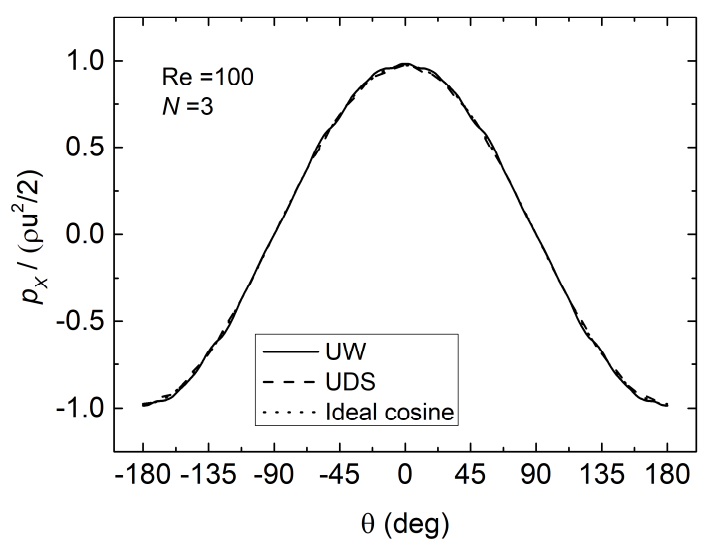

Fig.7. Output differential pressure $\left(p_{X}\right)$ at $\operatorname{Re}=100$, normalized to the dynamic pressure as a function of the wind direction for the UW and UDS solutions with $N=3$.
In order to estimate the performances of anemometers based on the proposed principle, we have considered two identical fluidic structures, indicated with $\mathrm{X}$ and $\mathrm{Y}$, respectively. The $\mathrm{X}$ structure is aligned as in Fig. 4(a), while the Y structure is simply rotated by $90^{\circ}$ with respect to section X. If $p_{X}(\theta, u)$ and $p_{Y}(\theta, u)$ are the differential pressures produced by section $\mathrm{X}$ and $\mathrm{Y}$, respectively, we obviously have:

$$
p_{Y}(\theta, u)=p_{X}(\pi / 2-\theta, u)=p_{X}(\theta-\pi / 2, u)
$$

If the approximation of the $p_{X}$ dependence on $\theta$ with a cosine function holds, then we can write:

$$
\begin{aligned}
& p_{X}(\theta, u)=p_{\text {MAX }}(u) \cos (\theta) \\
& p_{Y}(\theta, u)=p_{\text {MAX }}(u) \sin (\theta)
\end{aligned}
$$

Taking into account (32), estimates of angle $\theta$ (indicated with $\theta_{\mathrm{m}}$ ) and magnitude $p_{M A X}$ can be calculated by means of the following formulas:

$$
\begin{aligned}
& \theta_{m}=\arctan \left(p_{X}, p_{Y}\right) \\
& p_{M A X}=\sqrt{p_{X}^{2}+p_{Y}^{2}},
\end{aligned}
$$

where "arctan" is the four-quadrant inverse tangent function. The wind velocity $u$ can then be derived from $p_{M A X}$, since its dependence on the wind velocity is monotonic, as it will be shown later.

As an example, Fig. 8 shows the result of applying (33) and (34) to the case $\mathrm{Re}=100, N=1$ for both the UW and UDS configurations. The angular error, defined as $\theta-\theta_{\mathrm{m}}$, presents an important dependence on the wind direction $(\theta)$. Furthermore, as the wind direction is swept across the $360^{\circ}$ interval, $p_{M A X}$ shows oscillations that, for the examined case, reach $15 \%$ of the mean value. Both the angular error and the $p_{M A X}$ oscillations are due to the imperfect cosine approximation.
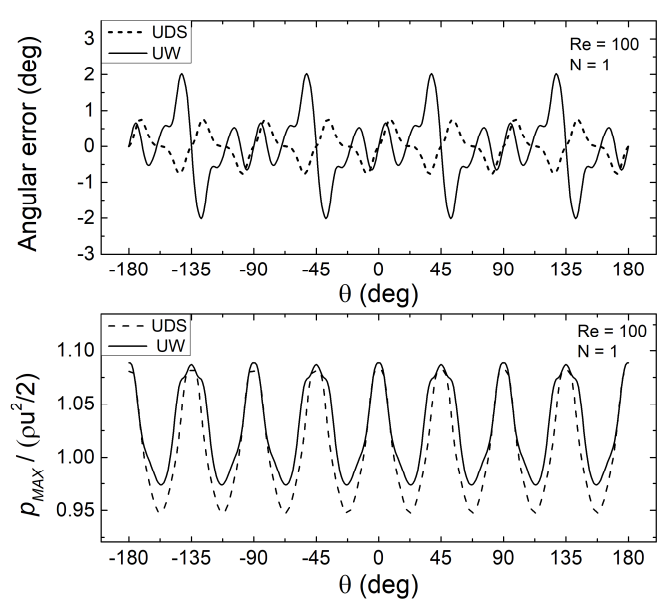

Fig. 8. Angular error and $p_{\text {MAX }}$ oscillations as a function of the wind direction.

Fig. 9 shows the $p_{M A X}$ over $\rho u^{2} / 2$ ratio, as a function of the Reynolds number for both the UDS and UW configurations with $N$ being varied from 1 to 5 . The $p_{M A X}$ value used in Fig.9 is averaged across the full $360^{\circ}$ wind direction range to filter 
out the oscillations.

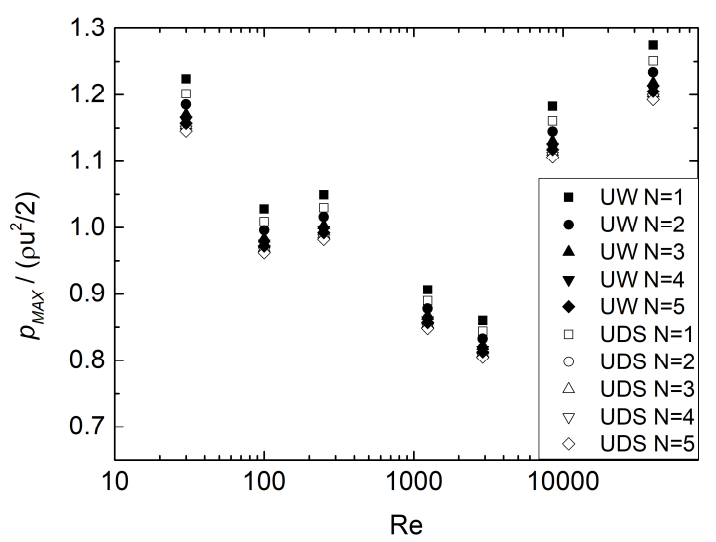

Fig. 9. Normalized $p_{M A X}$ values as function of Re for all configurations examined in this work. $p_{M A X}$ is averaged over a $360^{\circ}$ wind direction interval.

It is worth recalling that $p_{M A X}$ practically coincides with $B_{1}$, i.e. the fundamental component of the differential pressure $P_{X}$. The ratio $B_{1} / A_{1}$ falls within the interval $0.82 \pm 0.03$ in all examined cases, as Table II and III show for the UW (solution "a") and UDS configurations, respectively. As a consequence, the observed dependence of the $p_{M A X}$ over $\rho u^{2} / 2$ ratio on $\operatorname{Re}$ is mainly due to the dependence of the $A_{1}$ over $\rho u^{2} / 2$ ratio, which, in turn, is a general property of the flow.

Figure 10 shows the actual dependence of the differential pressure on the wind velocity for a particular cylinder diameter $(2 \mathrm{~cm})$ exposed to a room temperature $(300 \mathrm{~K})$ airflow. The air density and viscosity used to calculate the plot of Fig.10 are reported in the figure. All the examined cases (UW and UDS, $N$ from 1 to 5) produce plots that are practically undistinguishable in the scale of Fig.10, and are all represented by the solid line. As we have anticipated, the behavior is monotonic.

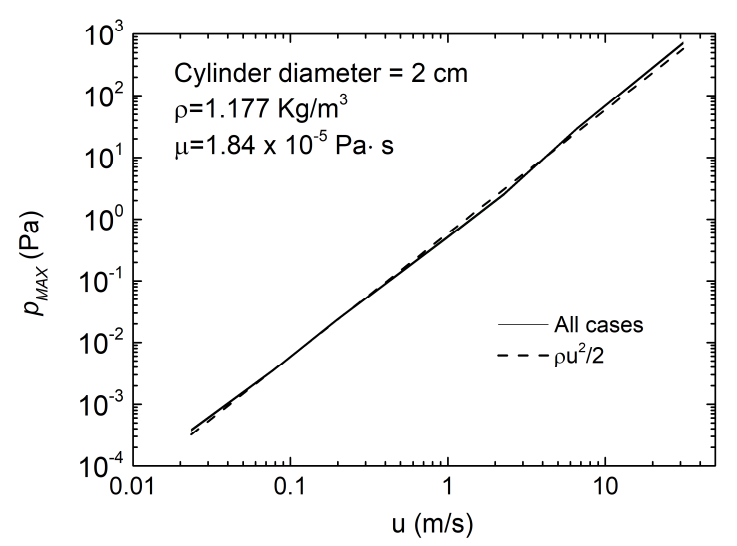

Fig.10. Example of estimated $p_{M A X}$ dependence on the wind velocity, obtained with a cylinder of $2 \mathrm{~cm}$ diameter, exposed to airstreams in standard conditions (see air parameters in the inset). In this scale, the curves obtained with UW and UDS structures with $\mathrm{N}$ from 1 to 5 are not distinguishable (solid line). The dashed line represent the dynamic pressure of the wind $\left(\rho u^{2} / 2\right)$.

The dashed line represents the dynamic pressure $\rho u^{2} / 2$.
Figure 10 suggests that we can approximate the $p_{M A X}$ behavior with the dynamic pressure and, consequently, use the following formula to estimate the wind velocity:

$u_{m}=\sqrt{\frac{2 P_{M A X}}{\rho}}$,

It can be shown that the maximum error deriving from this approximation is less than of $15 \%$ over the displayed velocity range. For better accuracy, a more complex fitting function can be adopted.

Finally, Fig.11(a) and (b) shows how the angular error and $p_{M A X}$ oscillation magnitude depend on the configuration (UW UDS) and number $N$. The angular error is the maximum absolute error obtained across the 360 degrees range.

Since both the angular error and $p_{M A X}$ oscillations depend also on $\operatorname{Re}$ (i.e. on the wind velocity), we have reported the best $(\min )$ and worst $(\max )$ value encountered when $\mathrm{Re}$ is swept from 30 to $40 \times 10^{3}$. It can be observed that, in all cases, the errors tend to decrease as $N$ is increased, although not in a monotonic fashion.

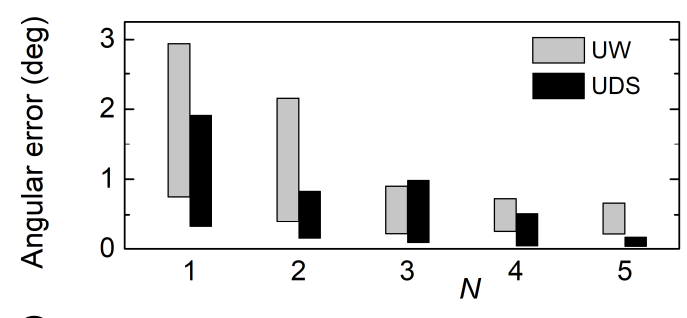

(a)

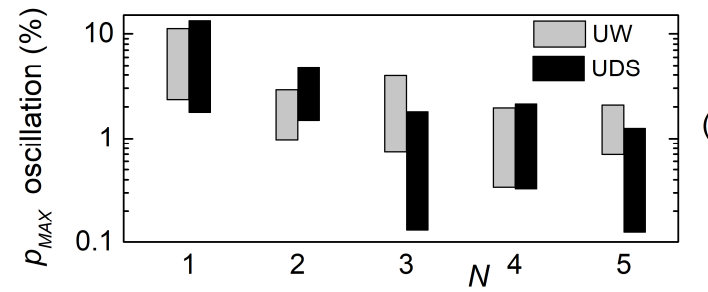

Fig.11. Angular error and $p_{M A X}$ oscillations as a function of $N$ for the UW and UDS configurations. The vertical bars span from the minimum to the maximum error estimated over the $30-40 \times 10^{3}$ Re range.

On the basis of the results of Fig.11, there is not a configuration which is clearly advantageous over the other. Overall, the UDS approach gives a slightly lower angular error, while magnitude oscillations are similar. Both configurations may be used to obtain an accurate estimate of the wind angle and velocity with magnitude oscillations smaller than $2.5 \%$ and angle errors less than $1^{\circ}$ for $N \geq 3$. The choice between the two configurations should rely on manufacturability issues.

\section{CLASSIFICATION AND ANALYSIS OF PREVIOUSLY PROPOSED DPA-BASED ANEMOMETERS}

An earlier work that contains some anticipations of the DPA approach is Ref. [31], where the non-monotonic angular behavior of a differential Pitot tube was solved by introducing more pressure ports distributed along a ring. Geometrical 
parameters were decided only by means of a trial and error procedure and only a qualitative explanation was proposed. In spite of the analogy between the ring and the cylinder, the device proposed in Ref. [31] does not fit within the categories proposed in this work, mainly because the wind direction is not perpendicular to the ring axis. Works describing anemometers that use the DPA approach as it is presented in this paper are reported and classified in Table IV.

TABLE IV. RECENT WORKS ON ANEMOMETERS BASED ON THE DPA APPROACH

\begin{tabular}{|l|l|l|l|l|l|}
\hline Year & Type & $\mathrm{N}$ & $\begin{array}{l}\text { Cylinder } \\
\text { diameter }\end{array}$ & $\begin{array}{l}\text { Channel } \\
\text { cross-section }\end{array}$ & $\begin{array}{l}\text { Angular } \\
\text { error }\end{array}$ \\
\hline $2009[22]$ & UW & 1 & $3 \mathrm{~cm}$ & $0.5 \times 1 \mathrm{~mm}^{2}$ & $\pm 6^{\circ}$ \\
\hline $2011[23]$ & UW & 2 & $2 \mathrm{~cm}$ & $1.0 \times 1.0 \mathrm{~mm}^{2}$ & $\pm 5^{\circ}$ \\
\hline $2016[24]$ & UDS & 3 & $2 \mathrm{~cm}$ & $1.0 \times 1.0 \mathrm{~mm}^{2}$ & $\pm 12^{\circ}$ \\
\hline
\end{tabular}

The directional anemometers proposed in [22] and [23] use uniform channel configurations with angles between the central diameter and the lateral ones that are practically coincident with the optimum angles proposed in Table I (solutions "a" for $N=1$ and $N=2$, respectively). Note that the criterion used to find the optimum angles in [22] and [23] was minimization of the mean square error (MSE) between the differential pressure and the cosine function. Optimization was performed at $\mathrm{Re}=2900$ through exhaustive exploration of all possible angle combinations. As in this work, digitized literature data [26] were used for the pressure distributions. The fact that such optimization, performed at a single Re value, produced an acceptable approximation of the cosine law across a large Re range was regarded as a pure experimental result and a clear explanation was missing.

In the light of the results of this work, the MSE optimization performed in [22] cancelled the third harmonics from the dependence on the wind direction, while the more complex structure of Ref. [23] cancelled both the third and the fifth harmonics. The excellent results that can be obtained even with a simple configuration as that of [22] is now explained by the dominant role played by the third harmonic (see sect.II).

The first example of UDS-DPA anemometer is the prototype proposed in [24], which was mounted on a small quadrotor to test the influence of the vehicle propellers on the wind measurement. That device was designed when the theory exposed in this work was being developed, thus the channel lengths were properly designed according to (28).

Considering the angular error reported in Table IV, it is apparent that increasing the $N$ value from one [22] to two [23] and three [24] did not produce the accuracy improvement predicted by Fig.11. It should be observed that the error estimates in Fig.11 do not take into account the errors introduced by the fluidic structures in the average computation. These errors may derive from (i) manufacturing uncertainties in the channel dimensions, (ii) non-fully developed flow in a significant portion of the channels, (iii) misalignment between the $\mathrm{X}$ and $\mathrm{Y}$ sections, (iv) perturbation of the pressure distribution produced by the channel openings on the cylinder lateral surface, whose dimensions coincide with the channel cross section (see table IV). All these mechanisms may have neutralized the theoretical accuracy improvements deriving from a larger $N$ value. Note that the worse performance of the UDS device is likely to derive from having experimented channels with aspect ratio close to $1: 1$, prone to non-developed flow effects. In all cases, important improvement can be expected to come from redesign of the fluidic structures in order to make them implement DPA with less non-idealities.

\section{CONCLUSIONS}

This work provides an analytical explanation for the DPA approach, a principle embodied in recently proposed directional anemometers. The possible DPA configurations have been classified according to an integer $N$, such that the number of diametric pressures involved in the average is $2 N+1$. Moreover, the interest has been restricted to two different classes of DPA configurations. In the first type, the diameter position ( $N$ angles) is free, but the average is performed with uniform weights (UW); the second type has free weights but uniform diameter spacing (UDS). It has been shown that, in the UW configurations, the $N$ angles can be chosen to cancel $N$ harmonics present in the diametric pressure dependence on the wind direction. Conversely, the $N$ weights of the UDS structures can be adjusted to obtain an approximation of the integral that extracts the first cosine term (fundamental one) from the diametric pressure. In both cases, the approximation of the target cosine dependence gets progressively better as $N$ is increased.

It has been shown that the fluidic structures used in the first two DPA anemometers [22,23] fit well in the classification proposed in this work, simply being example of UW configurations with $N=3$ and $N=5$, respectively. For both prototypes, the optimum angles were determined by means of a sort of blind numerical approach, which produced exactly the same result as the more general criterion proposed here. Using angular spectral analysis, this work also explains why the cosine approximation remains excellent across an impressively large interval of wind velocities.

The accuracy figures proposed in this work are to be regarded as lower bounds for the residual errors that can be obtained with the DPA technique. Manufacturing errors and non-ideal behavior of the fluidic structures are not considered. Nevertheless, the results proposed in this work should stimulate the design of new fluidic structures that perform DPA with better accuracy than the devices proposed so far, allowing fabrication of very accurate and inexpensive anemometers with compact size and low-power consumption.

\section{REFERENCES}

[1] T. A. Johansen, A. Cristofaro, K. Sørensen, J. M. Hansen, T. I. Fossen, "On estimation of wind velocity, angle-of-attack and sideslip angle of small UAVs using standard sensors", International Conference on Unmanned Aircraft Systems (ICUAS), Denver, Colorado, USA, June 9$12,2015$.

[2] M. Corno, S. Formentin, and S. M. Savaresi, "Data-Driven Online Speed Optimization in Autonomous Sailboats", IEEE Trans. on Intelligent Transportation Sys., vol. 17, no, 3, pp. 762-771, March 2016.

[3] D. Martínez, E. Clotet, M. Tresanchez, J. Moreno, J.M. Jiménez-Soto, R. Magrans, S. Marco, J. Palacín, "First characterization results obtained in a wind tunnel designed for indoor gas source detection", in proc. of the International Conference on Advanced Robotics (ICAR), July 27-31, 2015, Instambul, Turkey, 
[4] D. Han, S. Kim, S. Park, "Two-dimensional ultrasonic anemometer using the directivity angle of an ultrasonic sensor", Microelectronics Journal, vol. 39, pp. 1195-1199, March 2008.

[5] B. Allotta, L. Pugi, T. Massai, E. Boni, F. Guidi, M. Montagni, (2017, June). "Design and calibration of an innovative ultrasonic, arduino based anemometer" in proc. of. IEEE International conference on Environment and Electrical Engineering, June 6-9, 2017 Milan, Italy pp. 1-6.

[6] M. Iida, D. Kang, M. Taniwaki, M. Tanaka, and M. Umeda, "Localization of $\mathrm{CO}_{2}$ source by a hexapod robot equipped with an anemoscope and a gas sensor," Comput. Electron. Agriculture, vol. 63, pp. 73-80, Aug. 2008.

[7] L. Kowalski, M. T. Atienza, S. Gorreta, V. Jiménez, M. Domínguez-Pumar, S. Silvestre, Luis M. Castañer, "Spherical Wind Sensor for the Atmosphere of Mars", IEEE Sensors J., vol.16, no. 7, pp. 1887-1897, April 2016.

[8] K.A.A. Makinwa and J.H. Huijsing, "A wind-sensor interface using thermal sigma delta modulation techniques", Sensors and Actuators, A: Physical, vol.97-98, pp. 15-20, Apr. 2002.

[9] Y. Zhu, B. Chen, M. Qin, Q.-A. Huang, "2-D Micromachined Thermal Wind Sensors-A Review”, IEEE Internet Of Things J., vol.1, no.3, pp. 216-230, June 2014

[10] B. Idjeri, M. Laghrouche, and J. Boussey, "Wind Measurement Based on MEMS Micro-Anemometer With High Accuracy Using ANN Technique", IEEE Sensors J., vol.17, no. 13, pp. 4181-4188, July 2017.

[11] D. W. Bryer and R. C. Pankhurst, Pressure-Probe Methods for Determining Wind Speed and Flow Direction. St. Albans, U.K. Campfield Press, 1971, pp. 41-74.

[12] A. M. Judd, "Calibration of a five tube probe for measuring wind speed and direction", Journal of Physics E: Scientific Instruments, vol. 8, no 2 pp. 115-116, 1975

[13] I. C. Shepperd, "Mean flow measurements in three dimensions using a four hole pressure probe", 7th Australasian Hydraulics and Fluid Mechanics Conference, Brisbane, Australia, August 18-22, 1980, pp. 143-146.

[14] J. Chen, B. S. Haynes, D. F. Fletcher, "Cobra probe measurements of mean velocities, Reynolds stresses and higher-order velocity correlations in pipe flow", Experimental Thermal and Fluid Science, vol 21, no 4, pp. 206-217, 2000.

[15] D. P. Georgiou, K. F. Milidonis, "Fabrication and calibration of a subminiature 5-hole probe with embedded pressure sensors for use in extremely confined and complex flow areas in turbomachinery research facilities", Flow Measurement and Instrumentation, vol. 39, pp. 54-63, 2014

[16] J. L. Amick, G. C. Gill, "A stationary cylindrical wind speed and direction sensor for use in model testing, International research seminar on wind effects on buildings and structures", Ottawa, Canada, September 11-15, 1967 pp. 1-23.

[17] D. Surry, A. McDougall, I. Murley. "A miniature cylindrical wind speed and direction sensor", Journal of Wind Engineering and Industrial Aerodynamics, vol. 38, no 2, pp. 469-481 1991

[18] R. M. Eckman, R. J. Dobosy, D. L. Auble, T. W. Strong, T. L. Crawford, "A pressure-sphere anemometer for measuring turbulence and fluxes in hurricanes", J. of Atmospheric and Oceanic Technology, vol. 24, no 6, pp. 994-1007, June 2007.

[19] D. Telionis, Y. Yang, O. K. Rediniotis, "Recent developments in multihole probe (mhp) technology" 20th International Congress of Mechanical Engineering, Gramado, RS, Brazil November 15-20, 2009.

[20] Aeroprobe Online Product Catalog, Available at: http://www.aeroprobe.com/omniprobe/

[21] S. Shaw-Ward, A. Titchmarsh, D. M. Birch, "Calibration and use of n-hole velocity probes", AIAA Journal, vol. 53, no 2, pp. 336-346, 2014.

[22] P. Bruschi, M. Dei, M. Piotto, "A Low-Power 2-D Wind Sensor Based on Integrated Flow Meters”, IEEE Sens. J., vol. 9 no. 12, pp. 1688-1696, Dec. 2009.
[23] M.Piotto, G. Pennelli, P. Bruschi, "Fabrication and characterization of a directional anemometer based on a single chip MEMS flow sensor", Microelectronic Engineering, vol. 88, pp. 2214-2217, Nov. 2011.

[24] P. Bruschi, M. Piotto, F. Dell'Agnello, J. Ware, N. Roy, "Wind speed and direction detection by means of solid-state anemometers embedded on small quadcopters", Procedia Engineering vol. 168, pp. 802-805, Sept. 2016.

[25] Sensirion series SDP800 differential pressure sensors, Sensirion catalogue available on: https://www.sensirion.com.

[26] M. M. Zdravkovich, Flow Around Circular Cylinders: A Comprehensive Guide Through Flow Phenomena, Experiments, Applications, Mathematical Models and Computer Simulations..: Oxford Univ. Press, Oxford, U.K , 1997, vol. 1, pp. 1-109.

[27] F. Mayer, A. Häberly, H. Jacobs, G. Ofner, O. Paul, and H. Baltes, "Single chip CMOS anemometer," in Proc. Intern. Electron Devices Meeting, Washington, DC, Dec. 7, 1997, pp. 895-898.

[28] C. Liu, L. Du, Z. Zhao, "A directional cylindrical anemometer with four sets of differential pressure sensors", Rev. Sci. Instrum., vol. 87, pp. 035105-1-8, March 2016.

[29] T. Bourouina and J.-P. Grandchamp, "Modeling micropumps with electrical equivalent networks", J. Micromech. Microeng., vol. 6, n. 4, pp. 398-404, Dec. 1996.

[30] Scipy website: https://www.scipy.org/.

[31] W. A. Oost, "The Pressure Anemometer - an Instrument for Adverse Circumstances", J. Climate Appl. Meteorology, vol. 22. pp. 2075-2084, Dec. 1983.

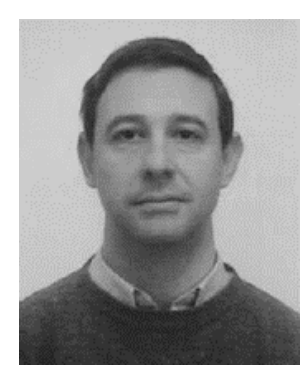

Paolo Bruschi was born in Massa, Italy, in 1964. He received the Laurea degree in Electronic Engineering from the University of Pisa, Italy, in 1989, and, in 1992, the Perfezionamento degree, equivalent to the $\mathrm{Ph} . \mathrm{D}$, from the S. Anna University School of Pisa. In 1993 he joined the Dipartimento di Ingegneria dell'Informazione of the University of Pisa, where he is currently an Associate Professor. His main areas of interest are the design of mixed signal integrated circuit and the development of MEMS sensors.

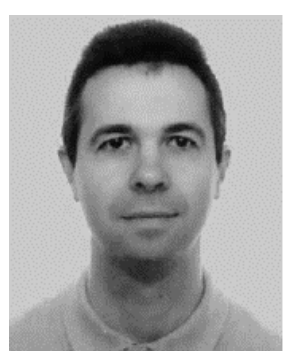

Massimo Piotto was born in 1970, La Spezia, Italy. He received his Laurea degree in Electronic Engineering from the University of Pisa, Italy, in 1996 and his Ph.D. degree in Electronic, Computer and Telecommunication Engineering in 2000. Since December 2001 he has been a researcher of the section of Pisa of the "Istituto di Elettronica e di Ingegneria dell'Informazione e delle Telecomunicazioni" - National Research Council (IEIITCNR). His main research interests concern MEMS-based smart sensors, microelectronic and nanoelectronic devices and technologies 